\title{
Comparison of Image Acquisition Techniques in Four-Dimensional Flow Cardiovascular MR on 3 Tesla in Volunteers and Tetralogy of Fallot Patients
}

\author{
Jun-Mei Zhang, Ru San Tan*, Shuo Zhang, Rob van der Geest, Pankaj Garg, Bao Ru Leong, Jennifer \\ Bryant, Tarinee Tangcharoen, Xiaodan Zhao, Ju Le Tan, Jos JM Westenberg, Liang Zhong
}

\begin{abstract}
Four-dimensional phase-contrast (PC) velocity-encoded flow magnetic resonance imaging (4D flow MRI) is a potentially valuable tool for studying cardiovascular hemodynamics for disease monitoring and/or treatment planning. In this study we compared the performance of two 4D flow MRI pulse sequences - echo-planar imaging (EPI) and segmented gradient-echo (turbo-field-echo or TFE on vendor's platform) - on a clinical 3T system in 6 human subjects including 3 patients with Tetralogy of Fallot (TOF). For aortic flow rate, the coefficients of variation $(\mathrm{COV})$ between $2 \mathrm{D}$ and 4D EPI were $7.0 \%$ and $7.7 \%$ for controls and patients respectively. The corresponding COV between 2D and 4D TFE were $19.0 \%$ and $18.3 \%$ for controls and patients respectively. The COV between 4D TFE and 4D EPI were larger than $18.7 \%$ in kinetic energy analysis. 4D EPI demonstrated acceptable accuracy of intra-cardiac flow quantification, which was also shown in the ex-vivo phantom measurements.
\end{abstract}

*Joint first author

Research supported by NMRC Grant (NMRC/BnB/0017/2015) and INITIATE project (NMRC/OFIRG/0018/2016).

Jun-Mei Zhang is with National Heart Research Institute Singapore, National Heart Center, Singapore, 5 Hospital Drive, Level 10, Singapore 169609 and Duke NUS Medical School Singapore (e-mail: zhang.junmei@nhcs.com.sg; zhangjunmei@gmail.com).

Ru San Tan is with Department of Cardiology, National Heart Center, Singapore, 5 Hospital Drive, Level 12, Singapore 169609 and Duke NUS Medical School Singapore (e-mail: tan.ru.san@nhcs.com.sg).

Shuo Zhang is with Philips Healthcare Singapore, 319762 (e-mail: zhang.shuo@philips.com).

Rob van der Geest is with Department of Radiology, Leiden University Medical Center, Leiden, the Netherlands (e-mail: R.J.van_der_Geest@lumc.nl).

Pankaj Garg is with Division of Biomedical Imaging, University of Leeds, Leeds, UK (e-mail: p.garg@leeds.ac.uk).

Leong Bao Ru is with Department of Nuclear Cardiac Imaging, National Heart Center, Singapore, 5 Hospital Drive, Level 12, Singapore 169609 (e-mail: leong.baoru@nhcs.com.sg)

Jennifer Bryant with National Heart Research Institute Singapore, National Heart Center, Singapore, 5 Hospital Drive, Level 12, Singapore 169609 (e-mail: bryant.jennifer.ann@nhcs.com.sg )

Tarinee Tangcharoen is with Ramathibodi Hospital, Bangkok, Thailand (e-mail: ttarinee@googlemail.com).

Xiaodan Zhao is with National Heart Research Institute Singapore, National Heart Center, Singapore, 5 Hospital Drive, Level 10, Singapore 169609 (e-mail: zhao.xiaodan@nhcs.com.sg).

Ju Le Tan is with Department of Cardiology, National Heart Center, Singapore, 5 Hospital Drive, Level 12, Singapore 169609 and Duke NUS Medical School Singapore (e-mail: tan.ju.le@nhcs.com.sg).

Jos JM Westenberg is with CardioVascular Imaging Group, Department of Radiology, Leiden University Medical Center, Leiden, The Netherlands (e-mail: J.J.M.Westenberg@lumc.nl)

Liang Zhong is with National Heart Research Institute Singapore, National Heart Center, Singapore, 5 Hospital Drive, Level 10, Singapore 169609 and Duke NUS Medical School Singapore (e-mail: zhong.liang@nhcs.com.sg).

\section{INTRODUCTION}

Traditional Cardiac MR (CMR) was valuable in identifying morphologic changes such as ventricular dilation and shape distortion[1] to signify dysfunction [2]. Two-dimensional (2D) velocity-encoded flow magnetic resonance imaging (MRI) is a well-established technique to quantify blood flow at particular acquisition plane [3]. Recently four-dimensional MRI (4D MRI) technique has opened a new era to acquire three-dimensional blood flow velocity information within the 3D domain [4][5][6][7].

To shorten the scan time, several data acceleration methods have become available. However comparisons of these 4D MRI sequences were limited. Garg et al. [8] have compared 3 sequences in phantoms and volunteers on 1.5 Tesla MR system. 4D EPI was reported to have highest consistency of intra-cardiac flow quantifications. We would like to test whether the same conclusion can be drawn in 3 Tesla (3T) MR system by comparing two RF-spoiled gradient-echo based pulse sequences, namely echo-planar imaging (EPI) and segmented gradient-echo (turbo-field-echo or TFE on vendor's platform) sequences.

Besides healthy volunteers, we extend the comparison to Tetralogy of Fallot (TOF) patients. TOF is a common congenital heart disease [9]. Surgical intervention and repair at desirable time is critical to reduce the mortality of TOF patients [10]. Applying 4D flow in TOF patients could potentially detect hemodynamic changes before ventricular morphological alterations [11]. Additional ex-vivo phantom measurements were also conducted with $3 \mathrm{~T}$ MRI system to further assess 4D EPI sequence.

\section{METHODS}

\section{A. Study Design and CMR Scans}

Three TOF patients ( 1 male, 2 females; aged $33 \pm 8$ years) and three controls ( 2 males, 1 female; aged $39 \pm 14$ years) were recruited. Informed consent was obtained before study participants underwent cardiac MRI on a clinical 3T system (Ingenia, Philips Healthcare, Best, the Netherlands).

For each participant, cardiac MRI was performed to determine cardiac morphology at standard planes: 2-, 3-, and 4-chamber long axis, as well as short-axis steady state free precession (SSFP) cine images, the latter covering both ventricles. Whole-heart 4D flow MRI was performed with free breathing without respiratory navigator gating. Measurements were acquired with volume coverage from the cardiac apex to aortic arch using RF-spoiled gradient-echo based TFE and EPI pulse sequences, with velocity encoding applied in three orthogonal directions. For both sequences, 
the spatial resolutions were identical (3.0-mm isotropic interpolated to $1.5-\mathrm{mm})$, while the combination of different under-sampling strategies in the phase-encoding dimension (partial Fourier), echo dimension (partial echo) and cardiac phase dimension (phase percentage) differed. A shot factor of 5 and 3 were used for 4D EPI and 4D TFE, respectively. Details of the imaging parameters are summarized in Table 1. In addition, standard 2D aortic flow MRI measurements were performed across the ascending aorta just distal to the coronary artery orifices, which served as a reference for comparison with 4D flow MRI results.

Table 1. Imaging parameters of 2D and 4D flow MRI using turbo-field-echo (TFE) and echo-planner (EPI).

\begin{tabular}{|l|c|c|c|}
\hline & $2 \mathrm{D}$ & $4 \mathrm{D}$ TFE & $4 \mathrm{D}$ EPI \\
\hline Field of view $(\mathrm{mm})$ & $320 \times 320$ & $340 \times 340$ & $340 \times 340$ \\
\hline Matrix size & $128 \times 117$ & $112 \times 111$ & $112 \times 111$ \\
\hline Number of slices & 1 & 67 & 67 \\
\hline Voxel size [Acquired] (mm) & $2.5 \times 2.6 \times 8$ & $3.0 \times 3.0 \times 3.0$ & $3.0 \times 3.0 \times 3.0$ \\
\hline $\begin{array}{l}\text { Voxel size [Reconstructed] } \\
(\mathrm{mm})\end{array}$ & $1.1 \times 1.1 \times 8$ & $1.5 \times 1.5 \times 1.5$ & $1.5 \times 1.5 \times 1.5$ \\
\hline TR / TE (ms) & $4.0-4.3 / 2.4-2.7$ & $3.3-4.0 / 1.8-2.9$ & $10 / 3.5$ \\
\hline Flip angle $\left({ }^{\circ}\right)$ & 10 & 10 & 10 \\
\hline Partial echo factor & no & 0.6 & no \\
\hline $\begin{array}{l}\text { Parallel imaging (SENSE) } \\
\text { factor }\end{array}$ & 2.2 to 2.4 & $2.6 \times 1.2$ & $2 \times 1$ \\
\hline Cardiac phases & 30 & 30 & 30 \\
\hline Cardiac phase percentage $(\%)$ & 60 & 60 & 100 \\
\hline VENC $(\mathrm{cm} / \mathrm{s})$ & 150 & 220 & 220 \\
\hline NSA & 1 & 1 & 1 \\
\hline Scan Time (min) & $0: 20-1: 30$ & $5-10$ & $5-10$ \\
\hline
\end{tabular}

NSA, number of signal averages; SENSE, sensitivity encoding; TE, echo time; TR, repetition time; VENC, encoding velocity

\section{B. Ex vivo Phantom Experiments}

Flow phantom experiments were carried out with steady flow of water with Gadolinium solution, in a straight tube with $1 \mathrm{~cm}$ diameter, submerged in a tank of water. ECG physiology simulation was used with 120 beats/min simulation retrospectively reconstructed into 5 phases. 2D PC and 4D flow MRI was performed with 3T Philips MR scanner.

\section{Image Processing and Analysis}

Post-acquisition analyses of 2D and 4D flow measurements were performed with the MASS software version 5.1 (Leiden University Medical Center, Leiden, the Netherlands). Intra-cardiac hemodynamics was qualitatively assessed from visualization of in-plane and through-plane velocities on cine images. From the 4D MRI data, aortic blood flow was quantified at a plane that corresponded exactly to that used for 2D aortic flow MRI measurement. For each subject, the endocardial contours of the left ventricle (LV) were drawn in the short axis cine image to facilitate the calculation of Kinetic Energy (KE). The definition of KE for a moving particle is shown in Eq. (1).

$$
K E=0.5 m v^{2}
$$

Here, $m$ and $v$ presents the mass and velocity of a moving particle, respectively. KE of flowing blood in LV can be computed by summing the $\mathrm{KE}$ of each individual voxel within the LV.

Continuous variables were reported as means \pm standard deviations. Comparisons between patients and controls were performed using two-sample t-test. All statistical tests were performed using SPSS.

\section{RESULTS AND DISCUSSION}

In general, 4D EPI allows for a better qualitative and quantitative representation of the complex cardiovascular hemodynamics. Fig. 1 shows the comparisons of 4D EPI and TFE on phase-contrast difference maps of the 4D flow measurement source images and streamline visualization of the flow pattern overlaid on the LVOT view at arbitrarily chosen cardiac phase. 4D EPI appeared to have less artifacts and flow distortions than 4D TFE, particularly in regions with lower velocity.

Aortic flow waveforms acquired with 2D, 4D EPI and 4D TFE flow MRI sequences in both patients and controls are displayed for comparison in Fig. 2. It is worth noting that phase offset errors of flow measurements with TFE sequences were corrected. In general, 4D EPI showed better agreement with 2D aortic flow MRI for both qualitative flow waveform representation and quantitative flow parameters such as peak aortic velocities and flow rates (Table 2). For aortic flow rate, the coefficients of variation (COV) between 2D and 4D EPI were $7.0 \%$ and $7.7 \%$ for controls and patients respectively (Table 2). 4D TFE measured lower net forward flow rates and higher peak velocity than those of 4D TFE. This could be explained by its lower temporal resolution (43 to $45 \mathrm{~ms}$ for TFE vs $40 \mathrm{~ms}$ for EPI) and incomplete echo sampling (partial echo factor 0.6) that easily led to phase dispersion and thus flow errors (as also demonstrated in Fig. 1). This was also in agreement with the recently work in both controlled phantom experiments and human scan $[8,13]$.

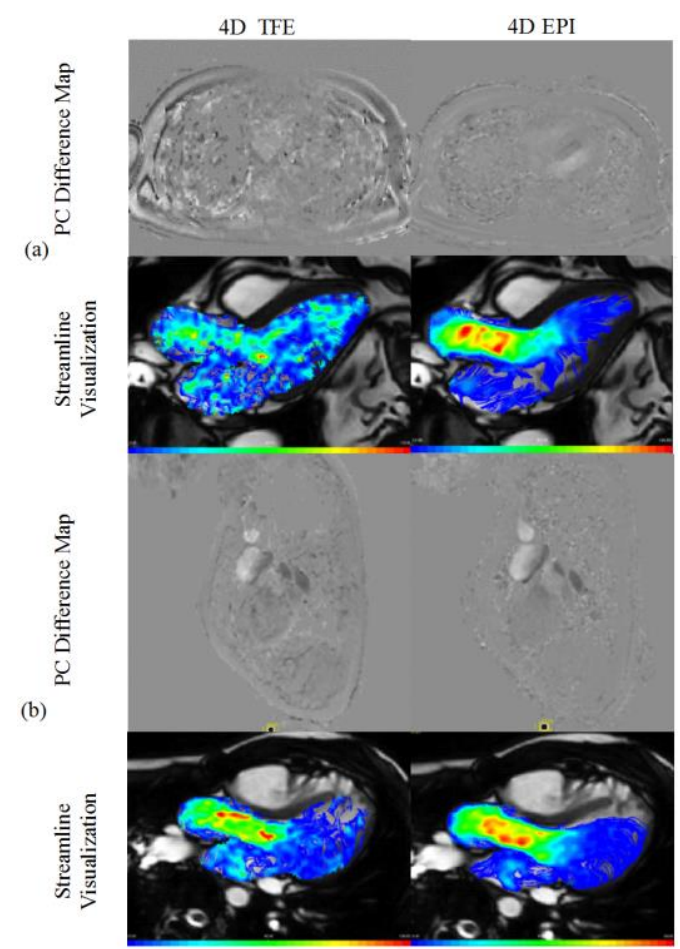

Figure 1. 4D flow MRI using TFE (left) and EPI (right) in Patient (a) and Control (b) as demonstrated with phase-contrast difference map (source images) in a quasi-3-chamber view in gray scale and streamline visulization in LVOT views. Blue: low velocity magnitude; red: high velocity magnitude 

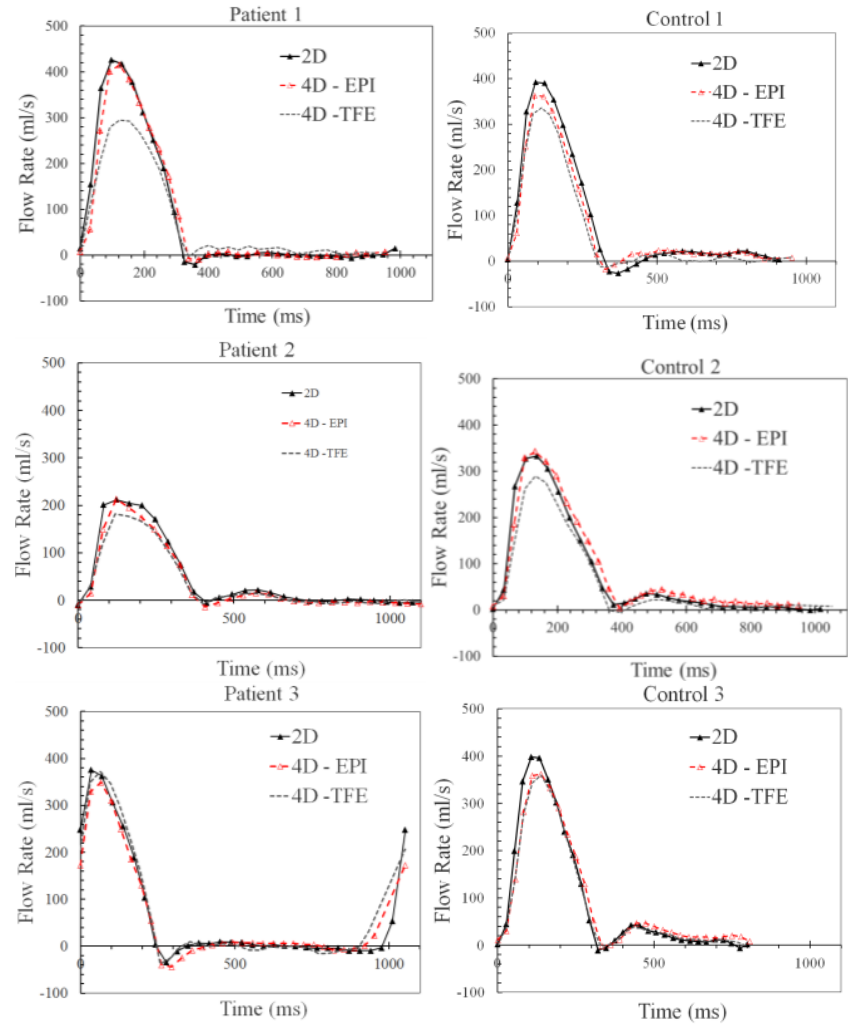

Figure 2. 4D aortic flow waveform of 3 patients (left) and 3 controls (right) obtained by 4D TFE and 4D EPI sequnces respectively in comparison to conventional 2D flow.

Table 2 Comparsion of aortic flow measurement (net forward flow $\mathrm{mL}$ and peak velocity $\mathrm{cm} / \mathrm{s}$ ) in controls and patients

\begin{tabular}{|c|c|c|c|c|c|}
\hline Controls & 2D & 4D EPI & COV1 (\%) & 4D TFE & COV2 (\%) \\
\hline $\begin{array}{c}\text { Net forward } \\
\text { flow (mL) }\end{array}$ & $78.6 \pm 1.2$ & $78.1 \pm 7.1$ & $7.0 \%$ & $65.0 \pm 5.3$ & $19.0 \%$ \\
\hline $\begin{array}{c}\text { Peak Velocity } \\
\text { (cm/s) }\end{array}$ & $81.4 \pm 8.0$ & $88.4 \pm 17.4$ & $12.9 \%$ & $\begin{array}{c}119.5 \pm 10 . \\
4\end{array}$ & $37.9 \%$ \\
\hline Patients & 2D & 4 D EPI & COV1 (\%) & $4 \mathrm{D}$ TFE & COV2 (\%) \\
\hline $\begin{array}{c}\text { Net forward } \\
\text { flow (mL) }\end{array}$ & $67.3 \pm 15.5$ & $62.3 \pm 19.2$ & $7.7 \%$ & $60.6 \pm 17.9$ & $18.3 \%$ \\
\hline $\begin{array}{c}\text { Peak Velocity } \\
(\mathrm{cm} / \mathrm{s})\end{array}$ & $101.3 \pm 34.4$ & $112.9 \pm 27.0$ & $11.1 \%$ & $\begin{array}{c}129.2 \pm 16 . \\
1\end{array}$ & $24.1 \%$ \\
\hline
\end{tabular}

COV1/ COV2: coefficients of variation between 2D and 4D EPI/ 4D TFE

Fig. 3 shows the KE waveforms derived from 4D EPI and 4D TFE data of both TOF patients and healthy volunteers. Similar to previous report [12], three distinct peaks in KE waveforms were observed for all subjects (both patients and controls), at systole, early diastole and late diastole.

Significant differences were found between 4D EPI and $4 \mathrm{D}$ TFE in the calculated KE paramters $(\geq 18.7 \%)$. Although the latter tended to underestimate flow rate (Fig. 2 and Table 2), the derived KE were higher than those from 4D EPI, as shown in Table 3. This may be explaned by the KE calculation method: in each voxel is linearly proportional to the second power of the velocity, which considers the magnitude and ignore the direction of the velocity vector. Due to artifacts and noise in 4D TFE scans, negative flow was detected in the left ventricular (LV) cavity region, as shown in Fig.4(c). This negative low resulted in a lower net flow rate and higher KE. This suggests that calculation of
KE may need to take into account the effect of the acquistion methods. Furthermore, assessment and interpretation of heart function using the current KE method should be done with caution.

Using 4D EPI sequences, the values of the peak systolic $\mathrm{KE}$ of controls $(1.29 \pm 0.32 \mathrm{~mJ})$ were close to the patients group $(2.29 \pm 1.47 \mathrm{~mJ}) \quad(\mathrm{p}=0.31)$. However, diastolic peak distolic KE were generally higher in TOF patients $(5.16 \pm 1.21$ $\mathrm{mJ})$ than those in controls $(1.97 \pm 0.22 \mathrm{~mJ})(\mathrm{p}=0.011)$. Normalized peak distaolic KE (by dividing it to LV end distolic volume (LVEDV)) was also higher in TOF patients $\left(0.044 \pm 0.019 \mathrm{~mJ} / \mathrm{m}^{3}\right)$ than that of controls $(0.017 \pm 0.0043$ $\left.\mathrm{mJ} / \mathrm{m}^{3}\right)(\mathrm{p}=0.066)$. The KE derived from 4D TFE sequences also showed similar characterristics of peak systolic and diastolic KE when comparing TOF patients with controls.

For ex vivo phantom measurement, the mean errors of flow rate at $21.25 \mathrm{ml} /$ beat and $16.67 \mathrm{ml} /$ beat were $0.78 \%$ and $1.3 \%$ for $2 \mathrm{D}$, and $7.2 \%$ and $4.4 \%$ for $4 \mathrm{D}$ flow based on EPI, respectively.
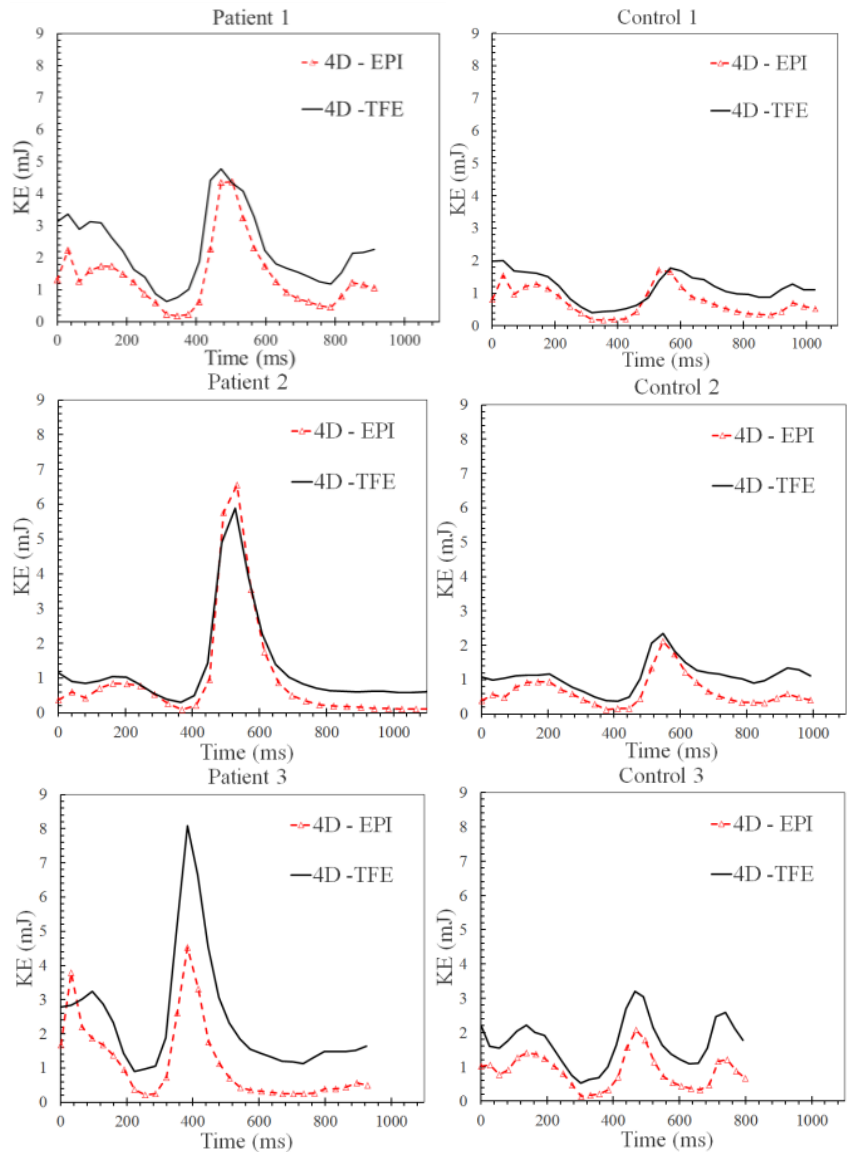

Figure 3. Kinetic energy (KE) in 3 patients (left) and 3 controls (right) derived from 4D flow MRI measurements with 4D EPI and 4D TFE sequences respectively. 
Table 3. Comparsions of KE and its related paramters between controls and patients

\begin{tabular}{|c|c|c|c|}
\hline Control & 4D EPI & 4D TFE & $\operatorname{COV}(\%)$ \\
\hline Average KE (mJ) & $0.76 \pm 0.11$ & $1.32 \pm 0.33$ & 53.1 \\
\hline Peak Systolic KE (mJ) & $1.29 \pm 0.32$ & $1.79 \pm 0.56$ & 31.0 \\
\hline Peak Diastolic KE (mJ) & $1.97 \pm 0.22$ & $2.44 \pm 0.72$ & 18.7 \\
\hline Average $\mathrm{KE} / \mathrm{BSA}\left(\mathrm{mJ} / \mathrm{m}^{2}\right)$ & $0.43 \pm 0.03$ & $0.75 \pm 0.13$ & 53.1 \\
\hline $\begin{array}{l}\text { Peak Systolic KE /BSA } \\
\left(\mathrm{mJ} / \mathrm{m}^{2}\right)\end{array}$ & $0.73 \pm 0.14$ & $1.00 \pm 0.24$ & 31.0 \\
\hline $\begin{array}{l}\text { Peak Diastolic KE/BSA } \\
\left(\mathrm{mJ} / \mathrm{m}^{2}\right)\end{array}$ & $1.13 \pm 0.19$ & $1.39 \pm 0.35$ & 18.7 \\
\hline $\begin{array}{l}\text { Average KE/LVEDV } \\
\left(\mathrm{mJ} / \mathrm{m}^{3}\right)\end{array}$ & $0.0063 \pm 0.00041$ & $0.011 \pm 0.001$ & 53.1 \\
\hline $\begin{array}{l}\text { Peak Systolic KE / LVEDV } \\
\left(\mathrm{mJ} / \mathrm{m}^{3}\right)\end{array}$ & $0.011 \pm 0.0019$ & $0.015 \pm 0.0024$ & 31.0 \\
\hline $\begin{array}{l}\text { Peak Diastolic KE/ LVEDV } \\
\left(\mathrm{mJ} / \mathrm{m}^{3}\right)\end{array}$ & $0.017 \pm 0.0043$ & $0.020 \pm 0.0047$ & 18.7 \\
\hline Patient & 4D EPI & 4D TFE & $\operatorname{COV}(\%)$ \\
\hline Average KE (mJ) & $1.15 \pm 0.25$ & $1.97 \pm 0.64$ & 49.6 \\
\hline Peak Systolic KE (mJ) & $2.29 \pm 1.47$ & $2.59 \pm 1.24$ & 28.7 \\
\hline Peak Diastolic KE (mJ) & $5.16 \pm 1.21$ & $6.25 \pm 1.69$ & 25.2 \\
\hline Average $\mathrm{KE} / \mathrm{BSA}\left(\mathrm{mJ} / \mathrm{m}^{2}\right)$ & $0.69 \pm 0.13$ & $1.16 \pm 0.27$ & 49.6 \\
\hline $\begin{array}{l}\text { Peak Systolic KE /BSA } \\
\left(\mathrm{mJ} / \mathrm{m}^{2}\right)\end{array}$ & $1.31 \pm 0.71$ & $1.50 \pm 0.63$ & 28.7 \\
\hline $\begin{array}{l}\text { Peak Diastolic KE /BSA } \\
\qquad\left(\mathrm{mJ} / \mathrm{m}^{2}\right)\end{array}$ & $3.16 \pm 1.16$ & $3.71 \pm 0.78$ & 25.2 \\
\hline $\begin{array}{l}\text { Average KE/LVEDV } \\
\qquad\left(\mathrm{mJ} / \mathrm{m}^{3}\right)\end{array}$ & $0.0094 \pm 0.00023$ & $0.016 \pm 0.0039$ & 49.6 \\
\hline $\begin{array}{l}\text { Peak Systolic KE / LVEDV } \\
\left(\mathrm{mJ} / \mathrm{m}^{3}\right)\end{array}$ & $0.019 \pm 0.012$ & $0.021 \pm 0.0081$ & 28.7 \\
\hline $\begin{array}{l}\text { Peak Diastolic KE/ LVEDV } \\
\left(\mathrm{mJ} / \mathrm{m}^{3}\right)\end{array}$ & $0.044 \pm 0.019$ & $0.053 \pm 0.018$ & 25.2 \\
\hline
\end{tabular}

BSA: body surface area; KE: Kinetic energy; COV: coefficients of variation between 4D EPI and 4D TFE

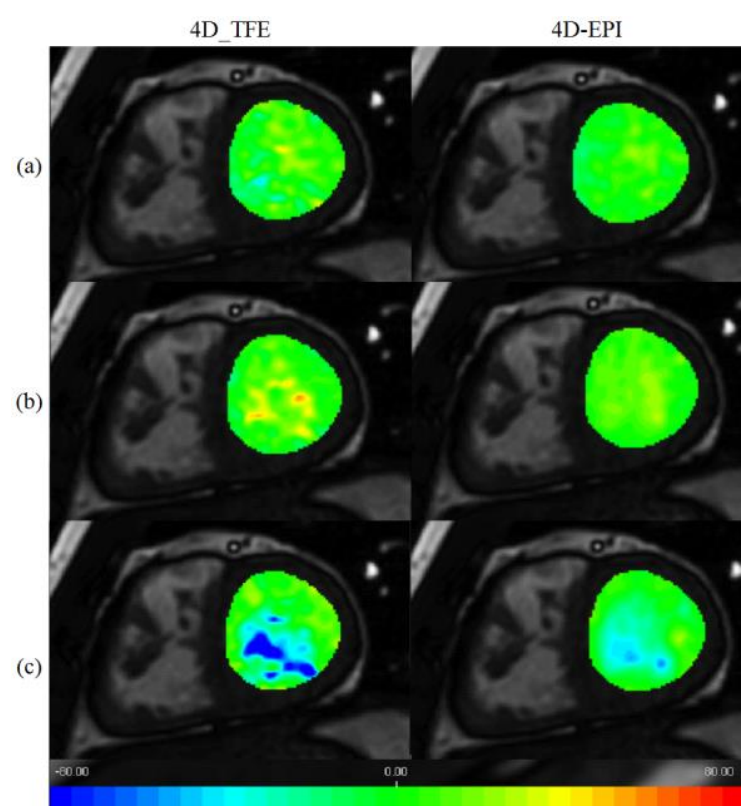

Figure 4. Intra-cardiac 4D flow in a patient with TOF using 4D TFE (left) and 4D EPI (right). Flow velocities were visualized in all three flow encoding directions: in-plane horizontal (a), in-plane vertical (b), and through plane (c). Images were selected at the diastolic phase from Patient 3.

\section{CONCLUSION}

We have shown that 4D flow MRI has potential value for the visualization and detection of abnormalities in complex cardiovascular hemodynamics in patients with TOF. For aortic flow rate, COV between $2 \mathrm{D}$ and $4 \mathrm{D}$ EPI were $7.0 \%$ and $7.7 \%$ for controls and patients respectively. The corresponding COV between 2D and 4D TFE were $19.0 \%$ and $18.3 \%$ for controls and patients respectively. The COV between 4D TFE and 4D EPI was larger than $18.7 \%$ in kinetic energy analysis. Clinical assessment and interpretation need to take into account the effects of the acquisition technique. Pre-data correction might be necessary for certain 4D MRI sequence such as 4D TFE. The sample size of this study is small. Further study with a larger cohort is needed to fully validate and standardize 4D flow measurements in these patient populations.

\section{ACKNOWLEDGMENT}

This research is supported by the INITIATE project (NMRC/OFIRG/0018/2016) and NMRC Grant (NMRC/BnB/0017/2015).

\section{REFERENCES}

[1] L. Zhong et al., "Right ventricular regional wall curvedness and area strain in patients with repaired tetralogy of Fallot," AJP Hear. Circ. Physiol., vol. 302, no. 6, pp. H1306-H1316, 2012.

[2] L. Adamson, H. a Vohra, and M. P. Haw, "Does pulmonary valve replacement post repair of tetralogy of Fallot improve right ventricular function?," Interact. Cardiovasc. Thorac. Surg., vol. 9, pp. 520-527, 2009.

[3] D. N. Firmin, G. L. Nayler, R. H. Klipstein, S. R. Underwood, R. S. Rees, and D. B. Longmore, "In vivo validation of MR velocity imaging," J. Comput. Assist. Tomogr., vol. 11, no. 5, pp. 751-756, 1987.

[4] M. Markl et al., "Time-resolved three-dimensional phase-contrast MRI," J. Magn. Reson. Imaging, vol. 17, no. 4, pp. 499-506, 2003. S. Nordmeyer et al., "Flow-sensitive four-dimensional cine magnetic resonance imaging for offline blood flow quantification in multiple vessels: A validation study," J. Magn. Reson. Imaging, vol. 32, no. 3, pp. 677-683, 2010.

[6] M. Mark1, W. Wallis, and A. Harloff, "Reproducibility of flow and wall shear stress analysis using flow-sensitive four-dimensional MRI," J. Magn. Reson. Imaging, vol. 33, no. 4, pp. 988-994, 2011. S. S. Vasanawala, K. Hanneman, M. T. Alley, and A. Hsiao, "Congenital heart disease assessment with 4D flow MRI," Journal of Magnetic Resonance Imaging, vol. 42, no. 4. pp. 870-886, 2015.

[8] P. Garg et al., "Comparison of fast acquisition strategies in whole-heart four-dimensional flow cardiac MR: Two-center, 1.5 Tesla, phantom and in vivo validation study," J. Magn. Reson. Imaging, vol. 47, no. 1, pp. 272-281, 2018.

[9] D. Lloyd-Jones et al., "Heart disease and stroke statistics-2010 update: A report from the american heart association," Circulation, vol. 121, no. 7. pp. e46-e215, 2010.

[10] J. P. Starr, "Tetralogy of Fallot: Yesterday and today," World J. Surg., vol. 34, no. 4, pp. 658-668, 2010.

[11] C. J. François et al., "4D cardiovascular magnetic resonance velocity mapping of alterations of right heart flow patterns and main pulmonary artery hemodynamics in tetralogy of Fallot," $J$. Cardiovasc. Magn. Reson., vol. 14, no. 1, 2012.

[12] M. Carlsson et al., "Quantification of left and right ventricular kinetic energy using four-dimensional intracardiac magnetic resonance imaging flow measurements," Am. J. Physiol. Heart Circ. Physiol., vol. 302, no. 4, pp. H893-900, 2012.

[13] S. Zhang, et al. "Whole-heart 4D phase-contrast MRI for clinical cardiovascular flow analysis: a comparison and validation on an imaging pulse sequence aspect," Proc. Int. Soc. Magn. Reson. Med., Paris, no. 4612, 2018. 